\title{
RESFRIAMENTO DO VERGALHÃO CA 60 NO PROCESSO DE LAMINAÇÃO A FRIO*
}

Claudine Guimarães Leite Cardoso ${ }^{1}$ Gilmar Aparecido da Silva ${ }^{2}$

\section{Resumo}

Em busca do aumento da produtividade em vergalhões é requerido o incremento de pesos das bobinas e velocidade no processo produtivo de laminação a frio do fio nervurado CA60 sendo notada a ocorrência de anomalias durante processamento de telas, a alteração de coloração na superfície do arame. Verificou-se a necessidade de um estudo para a caracterização do produto e modificação no processo decorrente das insistentes falhas nos processos posteriores durante a aplicação do fio prejudicando a produção esperada, assim como a ocorrência de sucata e redução de performance dos equipamentos. Com base na literatura, foram realizados ensaios para determinação de propriedades mecânicas e análise metalográfica cujos resultados se mostraram contrastantes nas amostras descrevendo comportamentos diferentes durante a produção do material. O projeto visa o entendimento do impacto gerado pelo aumento de velocidade de processamento minimizando as perdas de performance na trefilação por travamento de mancais de cassete e posteriores aplicações em ampliados.

Palavras-chave: Trefilação; Arame; Propriedades mecânicas; Textura.

\section{Abstract}

\section{COOLING WIRE CA 60 IN THE WIRE DRAWING PROCESS}

In search of increased productivity is required rebar in the increase of the coils and speed weights in the production of wire drawing process the wire cold ribbed CA60 being noticed anomalies occurring during processing of color change the screens on the surface of the wire. There was a need for a study to characterize the product and change in procedure due to repeated failures in subsequent processes during the implementation of wire harming the expected production, as well as the occurrence of scrap and equipment performance reduction. Based on the literature, experiments were performed to determine the mechanical properties and metallographic analysis whose results are shown in contrasting samples describing different behavior during production of the material. The design aims to understand the impact generated by an increase in processing speed performance while minimizing losses in the drawing for latching the cassette bearings and later welded mesh.

Keywords: Wire drawing; Wire; Mechanical properties; Texture.

1 Engenheira Metalúrgica, Mestrando do Mestrado Profissional em Materiais, Engenheira de Processo, Laminação a Frio, Votorantim Siderurgia; Centro Universitário de Volta Redonda (UniFOA), Barra Mansa, Rio de Janeiro e Brasil.

2 Administrador e Técnico em Eletromecânica, Técnico, Especialista de Processo, Laminação a Frio, Votorantim Siderurgia, Barra Mansa, Rio de Janeiro e Brasil. 


\section{INTRODUÇÃO}

A Máquina de telas da Votorantim Siderurgia apresentou paradas por embolo de arame transversal quando a barra é lançada e acertavam as guias na estação de soldagem, assim como os mancais de cassete dos laminadores a frio exibem alto índice de travamento quando empregados a velocidades de $16 \mathrm{~m} / \mathrm{s}$ na produção de bobinas de $2700 \mathrm{~kg}$.

No produto, foi observada a alteração da cor na superfície do CA 60 demonstrando aspecto amarelado sempre no centro das bobinas não sendo verificada nem na parte externa nem interna a mesma.

Foi notado o excesso de temperatura durante a formação da bobina baseado na energia dispersa pela conformação mecânica necessitando assim um desenvolvimento de resfriamento do vergalhão durante a laminação a frio. Analisando o processo constatou-se que a coloração sempre ocorre na parte da bobina com maior dificuldade de dissipar calor durante seu resfriamento em estoque, não podendo ser verificada durante o processo.

Caracterizado pelo trabalho a frio, ocorre na trefilação de arames o fenômeno mecânico-metalúrgico denominado encruamento com alteração das propriedades mecânicas do material. O encruamento é definido por Callister [1] como: "o aumento na dureza e resistência de um metal dúctil à medida que ele é submetido a uma deformação plástica abaixo da sua temperatura de recristalização". Estes materiais se deformam plasticamente pelo movimento das discordâncias, sendo a parte mais importante e difícil determinar como a densidade e distribuição das discordâncias variam com a deformação plástica pois estas são funções muito sensíveis da estrutura do cristal, energia de falha de empilhamento (EDE), temperatura e taxa de deformação.

O processo de deformação plástica é irreversível e a maior parcela do trabalho de deformação é transformada em calor. As tensões no estado final dependem do caminho seguido na deformação desenvolvendo uma orientação preferencial, ou textura, no qual certos planos cristalográficos tendem a se orientar de uma maneira preferencial com respeito à orientação de máxima deformação. No caso de arames, esse tipo de deformação tende a alinhar uma direção de cada cristal paralelamente ao eixo do arame.

O presente estudo detém como objetivo a caracterização do material laminado a frio em arame nervurado cuja composição química é pertinente ao aço ABNT 1008 para embasamento técnico e alteração do processo na usina da Votorantim Siderurgia localizada em Barra Mansa.

Diante do exposto, pretende-se mostrar os resultados obtidos dos sintomas através dos ensaios mecânicos e avaliações metalográficas além da alteração do resfriamento do fio visando corrigir os desvios que estão prejudicando a performance dos equipamentos.

\section{MATERIAIS E MÉTODOS}

A coleta de amostras foi realizada a partir do fio nervurado CA60 em aço ABNT 1008 após processamento em equipamento de trefilação EVG a velocidade de $14 \mathrm{~m} / \mathrm{s}$ no diâmetro de $3,8 \mathrm{~mm}$ na usina da Votorantim Siderurgia localizada em Barra Mansa/RJ. Manteve-se a sistemática ao posicionamento das amostras em relação ao volume de material enrolado na bobina nas diferenciadas posições descritas abaixo: 
A 1 - Amostra 1 - Externo da Bobina

A 2 - Amostra 2 - a $0,400 \mathrm{~kg}$ da bobina

A 3 - Amostra 3 - a $0,800 \mathrm{~kg}$ da bobina

A 4 - Amostra 4 - a 1,200 kg da bobina (centro da bobina)

O material apresenta composição química característica ao aço ABNT 1008 conforme verificado na tabela 1 determinada através de um espectrômetro Thermo Scientific $A R L^{\mathrm{TM}} 3460$ por emissão óptica na empresa Votorantim Siderurgia em Barra Mansa.

Tabela 1 - Análise química do material realizada através de espectrometria

\begin{tabular}{c|c|c|c|c|c|c|c|c|c}
\hline $\mathrm{C}(\%)$ & $\mathrm{Si}(\%)$ & $\mathrm{Mn}(\%)$ & $\mathrm{S}(\%)$ & $\mathrm{P}(\%)$ & $\mathrm{Cu}(\%)$ & $\mathrm{Ni}(\%)$ & $\mathrm{Cr}(\%)$ & $\mathrm{Sn}(\%)$ & $\mathrm{Mo}(\%)$ \\
\hline 0,07 & 0,11 & 0,45 & 0,019 & 0,017 & 0,264 & 0,073 & 0,049 & 0,023 & 0,022 \\
\hline
\end{tabular}

No ensaio da propriedade mecânica foi utilizado a máquina universal servo hidráulica da marca METROCON com uma célula de carga de $10 \mathrm{kN}$, avaliando a resistência à tração na Votorantim Siderurgia.

Para a metalografia em microscopia óptica e MEV/EBSD, realizada na Universidade Federal Fluminense (UFF), a etapa de lixamento foi realizada com seqüências de lixa 220 até papel de lixa metalográfica 1000. Na operação de polimento foi utilizado pano com pasta de diamante de $1 \mu \mathrm{m}$, seguida de alumina $1 \mu \mathrm{m}$ e sílica coloidal $0,02 \mu \mathrm{m}$ e posteriormente posicionadas no dissecador, evitando contaminação por umidade.

O ataque químico da superfície preparada foi realizado com a superfície polida sendo submetida uniformemente por imersão em nital (solução de ácido nítrico 3\% em álcool etílico) por 40 a 60 segundos, permitindo obter a imagem micrográfica. A micrografia é realizada observando que o nital não ataca a ferrita, mas delineia os contornos e escurece a perlita, se apresentando hachurada.

Através da análise com MEV/EBSD objetivou-se o estudo das orientações de grãos determinando a concentração ao redor de algumas orientações particulares determinando à forma como a rede cristalina desses grãos é arranjada espacialmente pelas componentes que representam orientações cristalinas no agrupamento de um razoável volume do material (número de grãos) por medidas das frações volumétricas de material a partir da intensidade que difratam o feixe de elétrons. Com essa finalidade, foram empregadas Função de Distribuição de Orientações Cristalinas (FDOC) segundo as coordenadas de Bunge.

A FDOC, é definida por 3 ângulos: $\varphi 1, \varphi 2$ e $\Phi$ constituindo três rotações consecutivas aplicadas aos eixos [100], [010] e [001] da célula cristalina do cristal. Através da metalografia é notada a quantidade de grãos de ferrita com cada orientação existente no policristal, representando a textura pela função de distribuição de orientações cristalinas (FDOC), figuras de pólo diretas e inversas.

$\mathrm{Na}$ caracterização da temperatura de processo foi utilizado a câmera termográfica FLIRi7 com sensibilidade térmica de $10^{\circ} \mathrm{C}$ e introduzido um fluxômetro no sistema de resfriamento dos cassetes para monitoramento da vazão de água disponibilizados na Votorantim Siderurgia. 


\section{RESULTADOS E DISCUSSÃO}

Pode-se verificar que o material ao ser desbobinado apresenta alterações nas propriedades mecânicas representadas pela queda no Limite de Resistência e aumento do Alongamento conforme tabela 2.

Tabela 2 - Resultado do ensaio de tração em amostras na direção de trefilação

\begin{tabular}{|c|c|c|c|c|c|c|c|c|c|}
\hline AMOSTRA & $\underset{(\mathrm{mm})}{0}$ & $\begin{array}{l}\text { AREA } \\
\text { SECAOO } \\
\left(\mathrm{mm}^{2}\right)\end{array}$ & $\begin{array}{l}\text { CARGA DE } \\
\text { ESCOAMENTO } \\
\text { (kgf) }\end{array}$ & $\begin{array}{l}\text { TENSĀO DE } \\
\text { ESCOAMENTO } \\
\left.\text { (kgf } \mathrm{mm}^{2}\right)\end{array}$ & $\begin{array}{c}\text { CARGA } \\
\text { DE } \\
\text { RUPTURA } \\
\text { (kgf) }\end{array}$ & $\begin{array}{c}\text { TENSĀO } \\
\text { DE } \\
\text { RUPTURA } \\
\left(\mathrm{kgf} / \mathrm{mm}^{2}\right)\end{array}$ & $\begin{array}{l}\text { RELAÇĀO } \\
\text { ELASTICA }\end{array}$ & $\begin{array}{c}\text { ALONGAMENTO } \\
(\%)\end{array}$ & $\begin{array}{l}\text { MASSA } \\
\text { LINEAR } \\
(\mathrm{kg} / \mathrm{m})\end{array}$ \\
\hline $\mathrm{A}_{1}$ & 3,8 & 11,34 & 0,87 & 76,7 & 0,98 & 86,4 & 1,13 & 6,6 & 0,089 \\
\hline $\mathrm{A} 2$ & 3,8 & 11,34 & 0,84 & 74,1 & 0,95 & 83,8 & 1,13 & 6,6 & 0,089 \\
\hline $\mathrm{A} 3$ & 3,78 & 11,21 & 0.81 & 72,2 & 0,92 & 82,1 & 1,14 & 7.9 & 0,088 \\
\hline A 4 & 3,78 & 11,21 & 0,83 & 74 & 0,92 & 82,1 & 1,11 & 7,9 & 0,088 \\
\hline
\end{tabular}

Através da análise metalográfica por microscopia óptica, essa alteração é explicada com a mudança na microestrutura, onde o material da superfície do arame não apresenta a textura alongada dos grãos característicos de processos de conformação a frio como mostrado na figura 1 pertinente a amostra 4.

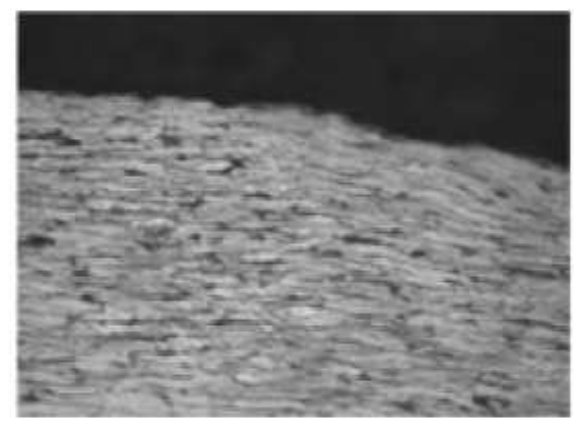

$500 x$

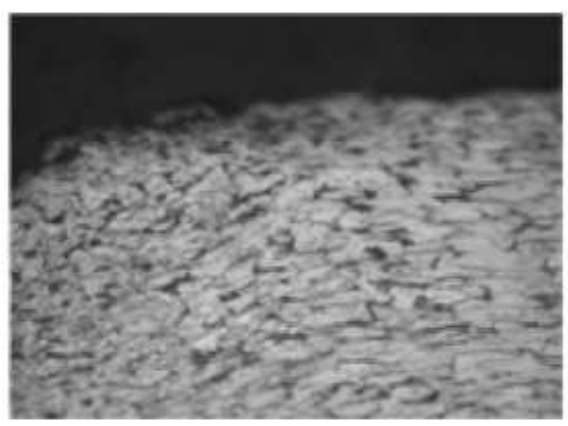

$500 x$

(a)

(b)

Figura 1 - Microscopia óptica (a) material característico em amostras referentes ao externo da bobina, (b) material de coloração alterada referente ao material da amostra 4 localizada o centro da bobina.

A análise metalográfica pelo MEV é demonstrada através da figura 2 por FDOC indicando a probabilidade de encontrar certa direção em um sistema da amostra nas seções de $\varphi 2=0^{\circ}$ a $\varphi 2=90^{\circ}$ em intervalos de $5^{\circ}$, percebendo picos da função correspondente apresentada nos tons avermelhados entre $\left(\varphi 1=0^{\circ}, \Phi=0^{\circ}\right)$ e $(\varphi 1=$ $\left.90^{\circ}, \Phi=0^{\circ}\right)$. Na amostra 1 foram analisados 1574 grãos podendo verificar pela FDOC que a maior intensidade de difração foi encontrado no sistema para o ábaco (9 3 13) $\left[\begin{array}{lll}0 & -1 & 1\end{array}\right]$ atingindo intensidade de difração de 35,4 para $\varphi 2=35^{\circ}$ na fibra DNO.

Já na amostra 4 foram analisados 498 grãos com dispersões entre os sistemas 68 7) [ 4 - -3 0] apresentando intensidade de difração de 19,2 a esquerda para $\varphi 2=30^{\circ}$; ( $\begin{aligned} & 0 \\ & 0\end{aligned}$ 1) [ [ - 14 0] com intensidade de difração de 9,2 superior a figura em $\varphi 1$; e (21 31 27) [5 -33 34] a direita em $\Phi$ na fibra DL demonstrando as diferentes orientações dos grãos para a amostra com coloração. 

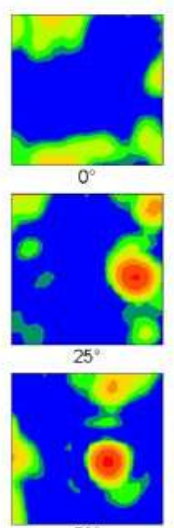

50

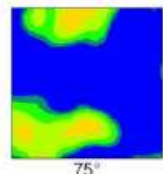

$75^{\circ}$
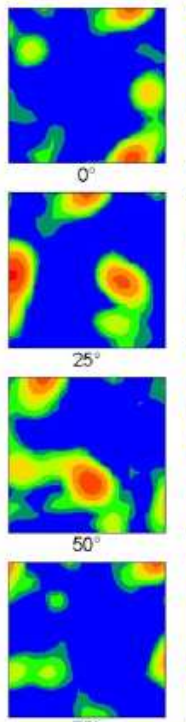

$75^{\circ}$
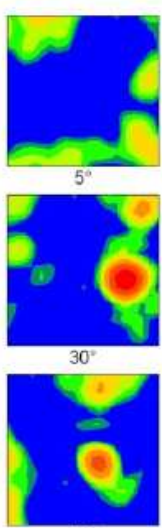

$55^{\circ}$

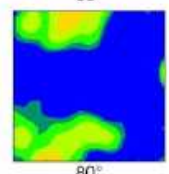

$80^{\circ}$
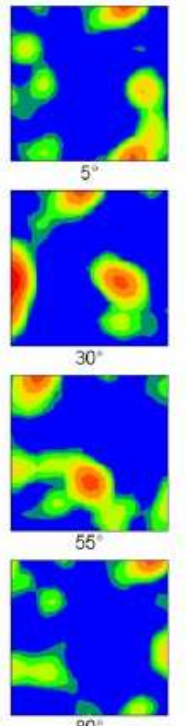

$80 "$
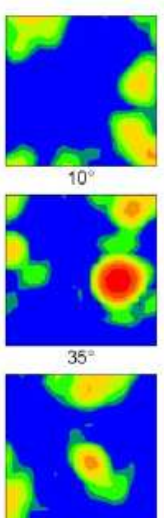

$60^{\circ}$
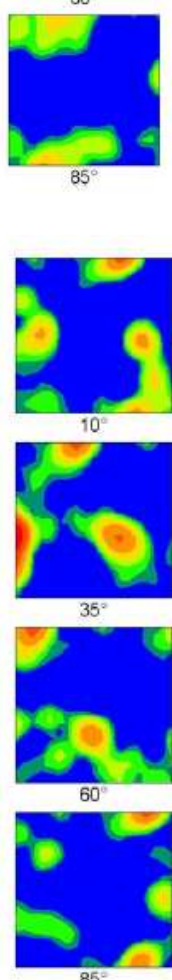

$85^{\circ}$
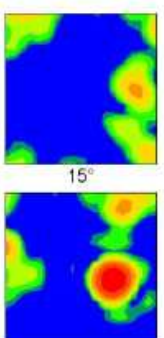

40

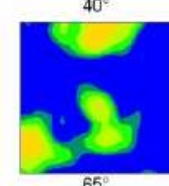

$65^{\circ}$

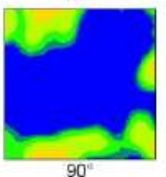

(a)
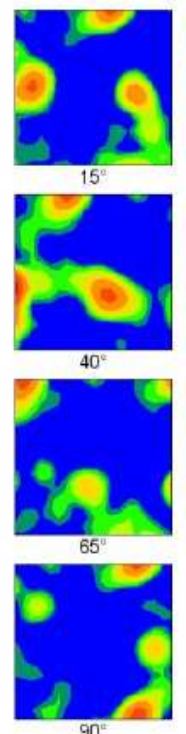

(b)
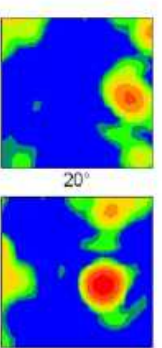

$45^{\circ}$

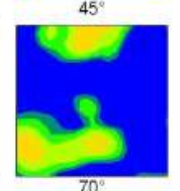

exture Name: Hamonic: $L=21, H W=5.0$

Calculation Method: Harmonic Series Expansion

Series Rank (): 21

Gaussian Smoothing: $50^{\circ}$

Sample Symmetry: Triclinic

Representation: Euler Angles (Bunge)

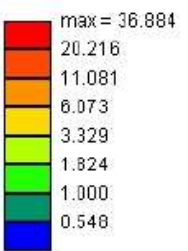

Constant Angle: $\varphi 2$

$\longrightarrow \varphi 1\left(0.0^{\circ}-90.0^{\circ}\right)$

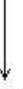

$\Phi\left(0.0^{\circ}-90.0^{\mathrm{m})}\right.$

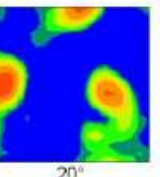

Texture Name: Harmonic: $L=21, H W=5.0$

Calculation Method: Harmonic Series Expansion

Serles Rank (1): 21

Gaussian Smoothing: $5.0^{\circ}$

Sample Symmetry: Triclinic

Representation: Euler Angles (Bunge)

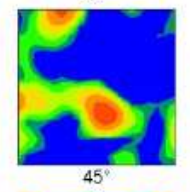

$\max =20.859$

12.572
7.577
4.567

4.567

2.753
1.659
1.000

1.000
0.603

Constant Angle: $\varphi 2$

$\longrightarrow \varphi 1\left(0.0^{\circ}-90.0^{\circ}\right)$

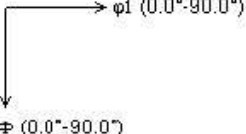

$\Phi\left(0.0^{*}-90.0\right)$

Figura 2 - Comparação da FDOC para ferrita: (a) amostra 1 externa a bobina; (b) amostra 4 com alteração de coloração.

As medições de temperatura foram realizadas através de termografia e demonstram a troca ineficiente de calor entre 0 arame e as bobinas do cabrestante destinadas ao seu resfriamento.

Conforme a figura $3 \mathrm{~b}$ a temperatura máxima na bobina foi de $184^{\circ} \mathrm{C}$ para a vazão de água de $7,781 / \mathrm{min}$ e diferença de temperatura da água de entrada e saída de $2,2^{\circ} \mathrm{C}$ antes da alteração proposta. Conforme orientações do fabricante, a temperatura máxima na aplicação dos mancais de cassetes é de $120^{\circ} \mathrm{C}$.

Pela figura $3 \mathrm{~d}$ pode-se notar a temperatura no final do processo atingindo $107^{\circ} \mathrm{C}$ na bobina após a melhoria implantada, com a redução correspondendo a $42 \%$ em temperatura para vazão de $15,75 \mathrm{I} / \mathrm{min}$ e diferença de temperatura da água de entrada e saída de $5,2^{\circ} \mathrm{C}$. 


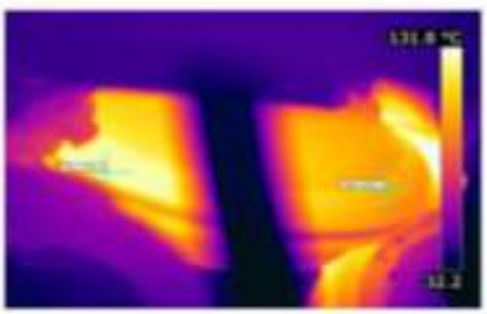

ANEL SUPERIOR

Maior Temperatura: $130^{\circ} \mathrm{C}$

Menor Temperatura: $100^{\circ} \mathrm{C}$

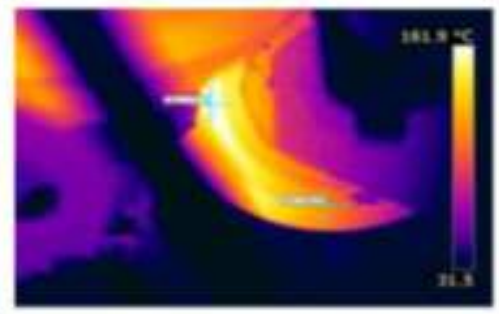

ANEL INFERIOR

Maior Temperatura: $172^{\circ} \mathrm{C}$

Menor Temperatura: $108^{\circ} \mathrm{C}$

(a) Temperatura nos anéis do cassete antes da modificação do processo
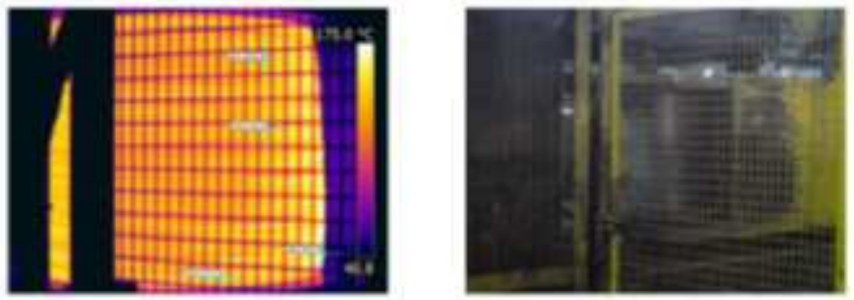

\section{BOBINA}

Maior Temperatura: $184^{\circ} \mathrm{C}$

Menor Temperatura: $161^{\circ} \mathrm{C}$

(b) Temperatura no bobinador vertical antes da modificação do processo

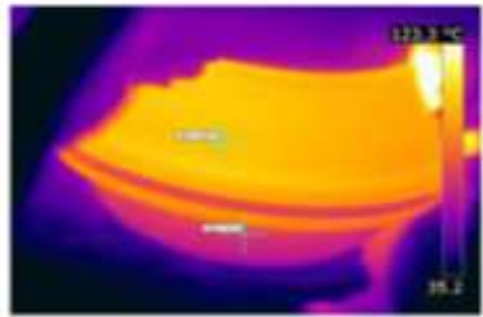

ANEL SUPERIOR

Maior Temperatura: $105^{\circ} \mathrm{C}$ Menor Temperatura: $73^{\circ} \mathrm{C}$

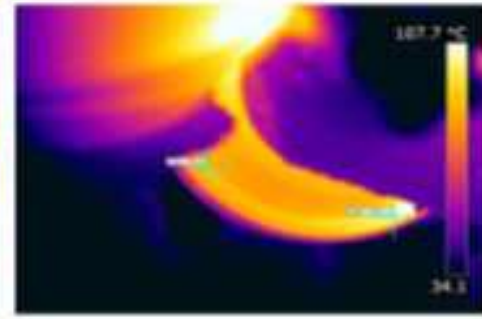

ANEL INFERIOR

Maior Temperatura: $102^{\circ} \mathrm{C}$

Menor Temperatura: $94^{\circ} \mathrm{C}$

(c) Temperatura nos anéis do cassete após modificação do processo
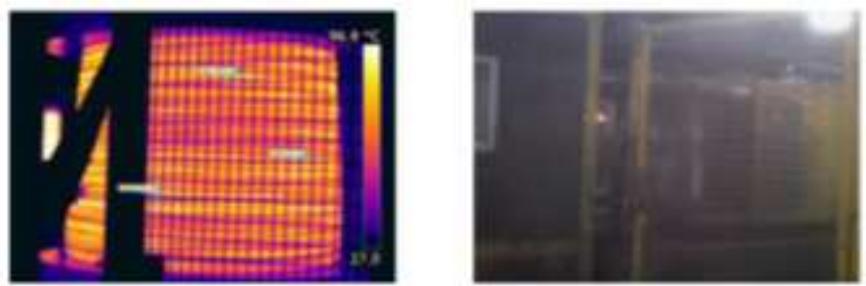

\section{BOBINA}

Maior Temperatura: $107^{\circ} \mathrm{C}$

Menor Temperatura: $97^{\circ} \mathrm{C}$

(d) Temperatura no bobinador vertical após modificação do processo

Figura 3 - Comparação de temperaturas durante o processo do laminador a frio sem a aplicação do projeto e com aplicação do resfriamento ao fio. 


\section{CONCLUSÃO}

A caracterização do material se mostrou com sucintas diferenças entre as amostras externas e internas da bobina que confirmam a heterogeneidade e interferência no ajuste operacional dos equipamentos. Entre estas medições foram constatadas diferença na resistência a tração e alongamento, respondidos com as alterações metalográficas.

Com isso, foram alterados os canais de refrigeração dos eixos dos cassetes e inserido ao processo um dispositivo em que o arame atravessa por dentro de um tubo com água, ambos retirando calor do fio e consequentemente a formação da bobina no carretel necessita de uma dispersão de calor menor que a existente anteriormente.

Pode-se notar a redução da temperatura na bobina de $42 \%$ considerando as medições do processo com e sem a aplicação do resfriamento ao fio nervurado CA 60.

\section{Agradecimentos}

À Votorantim Siderurgia pela oportunidade de demonstração da alteração do processo.

À UFF pela disponibilidade do equipamento de análise.

\section{REFERÊNCIAS}

1 CALLISTER, William D.,Jr; Ciência e Engenharia de Mateiais: Uma Introdução. Quinta Edição. Ed. LTC - Livros Técnicos e Científicos Editora S.A.. Rio de Janeiro. 2002. Trauzido por SOARES, Sérgio Murilo Stamile.

\section{BIBLIOGRAFIA}

1 VIANA, Carlos Sérgio da Costa. PAULA, Anderson dos Santos. Texturas de deformação. 30 f. Departamento de Engenharia Mecânica e Ciência dos Materiais Instituto Militar de Engenharia. Rio de Janeiro.

2 VIANA, Carlos Sérgio da Costa. LOPES, Andrea Machado. Texturas de transformação de fase. 136 f. Departamento de Engenharia Mecânica e Materiais - Instituto Militar de Engenharia. Rio de Janeiro.

3 PADILHA, Angelo Fernando; FILHO, Francisco Ambrozio. Técnicas de análise microestrutural. 1를. Edição. Ed. Hemus. Vol. 3, 4 e 5. 